\title{
OBSERVATIONS OF UNDERDENSE PLASMA LENS FOCUSING OF RELATIVISTIC ELECTRON BEAMS*
}

\author{
M.C. Thompson ${ }^{\dagger \ddagger}$, UCLA, Los Angeles, CA, 90095 and LLNL, Livermore, CA, 94550, USA \\ H. Badakov, J.B. Rosenzweig, G. Travish, UCLA, Los Angeles, CA, 90095, USA \\ R. Fliller, G. M. Kazakevich, P. Piot, J. Santucci, FNAL, Batavia, Illinois, 60510, USA \\ J. Li, R. Tikhoplav ${ }^{\S}$, University of Rochester, Rochester, New York 14627, USA
}

\begin{abstract}
Focusing of a $15 \mathrm{MeV}, 19 \mathrm{nC}$ electron bunch by an underdense plasma lens operated just beyond the threshold of the underdense condition has been demonstrated in experiments at the Fermilab NICADD Photoinjector Laboratory (FNPL). The strong $1.9 \mathrm{~cm}$ focal-length plasma-lens focused both transverse directions simultaneously and reduced the minimum area of the beam spot by a factor of 23 . Analysis of the beam-envelope evolution observed near the beam waist shows that the spherical aberrations of this underdense lens are lower than those of an overdense plasma lens, as predicted by theory. Correlations between the beam charge and the properties of the beam focus corroborate this conclusion.
\end{abstract}

\section{INTRODUCTION}

Plasma lenses are of great interest to the relativistic beam physics community because they can provide radially symmetric focusing gradients equivalent to a quadrupole lens gradient of the order $1 \mathrm{MT} / \mathrm{m}$, which exceeds the strength of conventional devices by many orders of magnitude [1, 2]. Additionally, it has been shown theoretically that adiabatic plasma lenses [3] can overcome the synchrotron radiation-induced limit on final focus spot size [4]. Consequently, there is great interest in using plasma lenses to achieve the small spot sizes and high luminosity necessary at the interaction point of future $e^{+} e^{-}$colliders such as the proposed International Linear Collider (ILC). We recently made the first observation of plasma lens focusing of a relativistic electron beam in the low-aberration underdense-regime: $n_{b} \gtrsim n_{p} / 2$, where $n_{b}$ and $n_{p}$ are the beam and plasma densities, respectively.

Plasma lenses operate in either the overdense $\left(n_{b}<<\right.$ $n_{p}$ ) or underdense regime. In the overdense case the plasma electrons spatially configure so that the plasma ions cancel the beam space charge and thus allow the beam to focus under its magnetic self-forces. These self-forces are not linear in distance from the beam axis $(r)$, or uniform in distance along the beam axis $(z)$, which leads to significant aberrations in the overdense case [2]. In the underdense

\footnotetext{
* Work supported by U.S. Dept. of Energy Contracts No. DE-FG0392ER40693 and W-7405-ENG-48

$\dagger$ dr.mcthompson@gmail.com

$\ddagger$ Now at TAE Inc., Irvine, CA

$\S$ Now at UCLA
}

case the strong electric field created by the space charge of the electron beam ejects the plasma electrons from the beam region entirely, leaving a uniform ion column. It can be shown that the radial-focusing electric-force of this ion column is linear and given by $F_{\text {radial }}=-2 \pi n_{p} e^{2} r$, where $e$ is the electron charge. An underdense plasma lens is, therefore, theoretically free of spherical aberration in the limit that the ion column is as wide as the beam and the ions are immobile. The head of the beam is not focused in an underdense plasma lens because of the finite response time of the plasma electron density distribution. Focusing by overdense plasma lenses of up to $4 \mathrm{MT} / \mathrm{m}$ strength has been demonstrated in previous experiments [5 - 9]. Lensconfiguration focusing in the low-aberration underdenseplasma-lens regime, however, has not been previously observed.

\section{EXPERIMENTAL METHODOLOGY}

In the experiment, a $14.8 \mathrm{MeV}$ electron beam is focused by a plasma lens of approximately gaussian profile in $z$ with FWHM of $19.3 \mathrm{~mm}$ and peak density $n_{\text {, peak }}=4.9$ $\mathrm{x} 10^{12} \mathrm{~cm}^{-3}$. This plasma lens is uniform in $r$ on the length scale of the beam, has an average focusing strength $K=2561 \mathrm{~m}^{-2}$ over an effective length of $l=20.5 \mathrm{~mm}$, and a focal length $(f=1 / K l)$ of $1.9 \mathrm{~cm}$. In magnetic quadrupole units, the lens has an average focusing gradient of $126 \mathrm{~T} / \mathrm{m}$, which is about 40 times stronger than the magnetic qudrupole fields used to transport the beam to our experiment.

The plasma lens was created using a direct-current

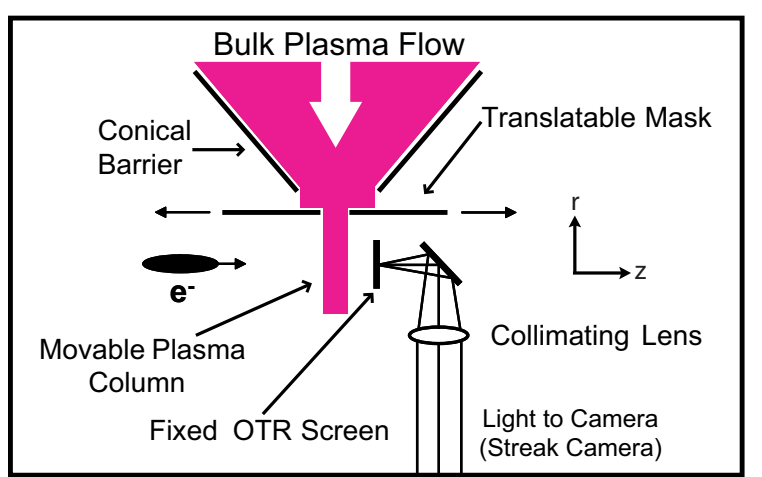

Figure 1: Schematic of the plasma lens apparatus. 
electrical-discharge transversely offset from the beam orbit as illustrated in Fig. 1. A bulk plasma column of approximate width $5 \mathrm{~cm}$ FWHM is produced using our pulsed argon-discharge plasma-source [10] and allowed to diffuse toward the beam path under the weak confinement of a 53 $\mathrm{G}$ solenoidal magnetic field aligned with the plasma flow. A conical stainless steel barrier suppresses diffusion into the interaction area and a thin slice of the static plasma column is selected using a translatable mask with a $1.25 \mathrm{~cm}$ wide slit. This arrangement allows the distance between the plasma lens column and the fixed optical transition radiation (OTR) diagnostic screen to be varied without altering the OTR collection optics. An in-vacuum, $160 \mathrm{~mm}$ focal length, 2 inch diameter lens provides quasi-parallel collimation of the OTR produced when the electron beam emerges from the back of a polished aluminum foil. The OTR is then transported either $30 \mathrm{~cm}$ to a conventional CCD camera or $7 \mathrm{~m}$ to a streak camera where the light is imaged onto the streak slit using a single $280 \mathrm{~mm}$ focal length lens.

The electron beam for the experiment is provided by the Fermilab NICADD Photoinjector Laboratory (FNPL) facility [11]. The FNPL injector is a $15 \mathrm{MeV}$ electron source consisting of a normal conducting L-band RF gun with a $\mathrm{Cs}_{2} \mathrm{Te}$ photo-cathode and a 9-cell superconducting postaccelerator cavity. After acceleration, the electron beam is propagated at a tight focus through a $10 \mu \mathrm{m}$ thick aluminum vacuum-isolation foil and refocused into the plasma lens experiment. Scattering in the aluminum foil significantly increases the beam emittance. The beam is transported $2.2 \mathrm{~m}$ from the vacuum foil to the center of the
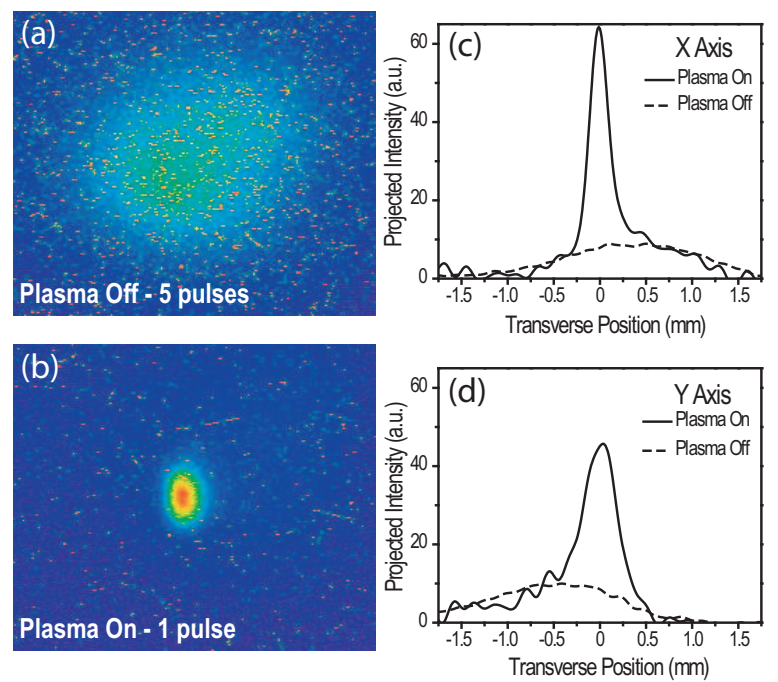

Figure 2: Images of the unfocused (a) and focused (b) electron beam OTR displayed with the same scaled to intensity color map. In order to provide sufficient contrast, the unfocused image (a) is the integration of 5 beam pulses. The projected intensity of the focused and unfocused beams (normalized to 1 pulse) in the $\mathrm{x}$ axis (c) and $\mathrm{y}$ axis (d) is also shown. plasma lens.

\section{UNDERDENSE PLASMA FOCUSING}

In order to understand the spherical aberrations of our plasma lens we made a series of round beam focusing measurements with different spacings between the plasma lens and OTR screen. These experiments used a 14.8 $\mathrm{MeV}$ bi-gaussian electron beam with initial dimensions $\sigma_{x}$ $=692 \pm 54 \mu \mathrm{m}, \sigma_{y}=656 \pm 51 \mu \mathrm{m}, \mathrm{Q}=18.8 \pm 2.0 \mathrm{nC}$, and $\sigma_{t}=22 \pm 3 \mathrm{ps}$. Consequently the initial peak beam density was $n_{\text {b, peak }}=2.5 \pm 0.5 \times 10^{12} \mathrm{~cm}^{-3}$. Uncertainties are dominated by shot-to-shot variation of the beam. Peak density of the plasma lens is $n_{p, p e a k} \simeq 4.9 \times 10^{12} \mathrm{~cm}^{-3}$ so $n_{\text {beak }} \approx n_{\text {p,peak }} / 2$ putting the experiment just on the boundary of the underdense regime, in terms of beam and plasma peak densities, at the entrance to the plasma lens. This is a lower bound however since the beam is substantially denser compared to the edge of the gaussian plasma column which it first encounters and, since the lens is thick, both analytic calculations and simulations show the beam core has compressed somewhat by the time it reaches the center of the plasma lens. We can, therefore, unequivocally state that the beam focusing is governed by underdense dynamics at the threshold of the regime. When optimally focused, a beam waist with $x_{\mathrm{FWHM}}=259 \pm 22 \mu \mathrm{m}$ and $y_{\mathrm{FWHM}}$ $=423 \pm 41 \mu \mathrm{m}$ is achieved as shown in Fig. 2 . The demagnification factor of 6.29 in $x$ and 3.65 in $y$ means that the transverse area of the focused beam is reduced by a factor of 23. Note that the overall distribution of the plasma focused beam observed on OTR is not a single gaussian but a superposition of the strongly focused core and unfocused head and tail of beam, each of which remains roughly gaussian. By using the FWHM to characterize the focused beam the we automatically exclude the unfocused portions of the beam (halo) which are visible as broad bases of the focused peaks in Fig. 2(c) and Fig. 2(d).

The effect of linear transverse focusing elements, whether plasma or magnetic, on an electron beam traveling in the $z$ direction can be modeled (neglecting space charge effects) by the beam envelope equation:

$$
\frac{d^{2} \sigma_{x}}{d z^{2}}+K \sigma_{x}=\frac{\epsilon_{x}^{2}}{\sigma_{x}^{3}},
$$

where $\sigma_{x}$ is the rms beam size in $x, \epsilon_{x}$ is the geometric beam emittance in $x$, and $K$ is the focusing strength of the lens. The equivalent equation for the other transverse direction can be written by substituting $y$ for $x$. For an underdense plasma lens $K=2 \pi r_{e} n_{p} / \gamma$, where $r_{e}$ is the classical electron radius, and $\gamma$ is the Lorentz factor [12]. Detailed predictions of the plasma lens focusing of the beam core can be made by solving Eq. 1 using a function $n_{p}(z)$ that describes the shape of our thick plasma lens. The envelope equation can also be use to describe the focusing of lenses with aberrations. A general way to do this is to define an effective emittance $\epsilon_{\text {eff }}=\sqrt{\epsilon_{0}^{2}+\beta_{0} \epsilon_{0} \delta \theta^{2}}$ for use in Eq. 1 which includes both the original emittance 


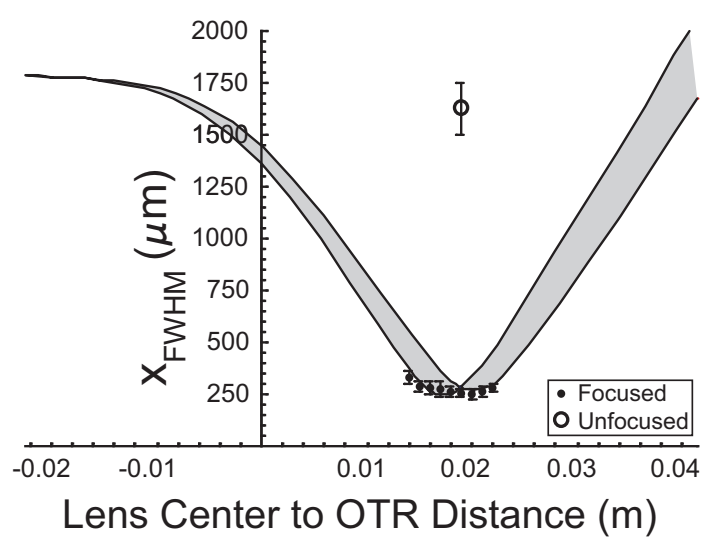

Figure 3: Measurements of underdense plasma lens focusing of a round beam in the $\mathrm{x}$ axis shown with solutions to the envelope equation (solid lines and shaded region). The shaded region indicate the shift in focal length caused by the variation in plasma lens parameters.

of the beam $\epsilon_{0}$ and the extra angular spread resulting from the lens aberrations $\delta \theta$ [13]. The aberration induced angular spread can be defined as $\delta \theta=\left(\sqrt{\beta_{0} \epsilon_{0}} / f\right)(\Delta K / K)$ where $\beta_{0}=\left(\sigma_{0}^{2} / \epsilon_{0}\right)$ is the beam beta-function at the lens entrance, $f$ is the lens focal length, and $\Delta K$ is the rms amount the lens strength deviates from linear expectation over the beam. Su et al. have shown that the minimum spherical aberration of an overdense plasma lens focusing a gaussian beam is $\Delta K / K=0.21$ [2]. As stated previously, an underdense plasma lens can theoretically be spherical aberration free $(\Delta K / K=0)$ in the ideal limit.

Fitting the predictions of the envelope equation to the focusing data obtained in the round beam case described above, Fig. 3, reveals a great deal about the plasma lens and its aberrations. The unfocused beam envelope is calculated using the initial conditions at the vacuum foil. The plasma lens parameters vary somewhat as the mask is translated, which leads to a slight variation in the lens focal length and, consequently, a broadening of the observed depth of focus as shown in Fig. 3. While the focal length is determined by the known plasma lens parameters, the size of the beam waist is determined by the parameter $\epsilon_{e f f}$ which was obtained by a best fit to the data. For example, in the $x$ direction the curve fit shown in Fig. 3 gives $\epsilon_{e f f, x, n}=110 \mathrm{~mm}$-mrad, where we have followed convention and used normalized emittance $\epsilon_{n}=\beta \gamma \epsilon$. A value of $\epsilon_{x, n}=87 \mathrm{~mm}$-mrad was measured by a quadrupole scan downstream of the vacuum isolation foil. Using the above expression for $\epsilon_{\text {eff }}$ we can immediately calculate $\Delta K_{x} / K_{x}=0.076 \pm 0.006$ which is well below the overdense lens minimum of 0.21 and thus strongly indicative of underdense operation. The observed saturation of the focused beam spot size as the beam charge is increased to the expected underdense threshold, Fig. 4, also supports the case for underdense operation.

In conclusion, we have measured extreme demagnifica-

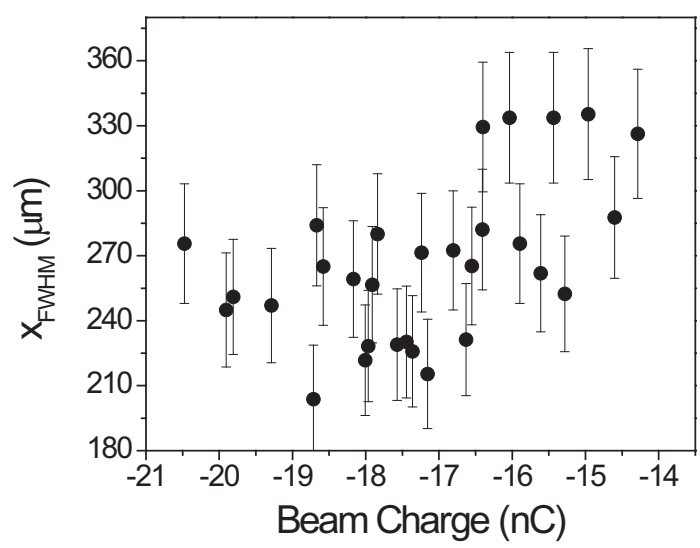

Figure 4: Correlation between the beam charge and the focused beam spot size.

tion of a high brightness electron beam by a strong underdense plasma lens and shown that, as predicted, this lens has lower aberrations than overdense lenses even near the boundary of the underdense regime $\left(n_{b} \gtrsim n_{p} / 2\right)$. We conjecture that the lens has a reasonably well formed ion column in this threshold regime due to the additive effect of the electrostatic forces and the mutual repulsion between the beam and plasma return currents. We are exploring the validity of this explanation through simulation. Operation at the boundary of the underdense regime may be an attractive plasma lens scenario since it combines low aberration with minimal beam density. Lowering $n_{b}$ may be one way to mitigate the problem of ion motion [14] for ILC class beams. A full analysis of this underdense plasma lens experiment, including our time-resolved beam measurements, will be reported elsewhere [15].

\section{REFERENCES}

[1] P. Chen, Part. Accel. 20, 171-182 (1987).

[2] J. J. Su, et al., Phys. Rev. A 41, 3321 (1990).

[3] P. Chen, et al., Phys. Rev. Lett. 64, 1231 (1990).

[4] K. Oide, Phys. Rev. Lett. 61, 1713-1715 (1988).

[5] H. Nakanishi, et al., Phys. Rev. Lett. 66, 1870 (1991).

[6] G. Hairapetian, et al., Phys. Rev. Lett. 72, 2403 (1994).

[7] R. Govil, et al., Phys. Rev. Lett. 83, 3202 (1999).

[8] P. R. Bolton for the E150 Collaboration, Int. J. Mod. Phys. A 18, 2843 (2003).

[9] J. S. T. Ng, et al., Phys. Rev. Lett. 87, 244801 (2001).

[10] M. C. Thompson, J. B. Rosenzweig, and G. Travish, Rev. Sci. Instrum. 76, 013303 (2005).

[11] J.-P. Carneiro, et al., "First Results of the Fermilab HighBrightness RF Photoinjector," in Proc. PAC 1999, IEEE, 1999, p. 2027.

[12] N. Barov, and J. B. Rosenzweig, Phys. Rev. E 49, 4407 (1994).

[13] J. B. Rosenzweig, et al., Part. Accel. 24, 11 (1988).

[14] J. B. Rosenzweig, et al., Phys. Rev. Lett. 95, 195002 (2005).

[15] M. C. Thompson, et al., Phys. Plasmas, (to be submitted). 